\title{
ON THE SCATTERING AMPLITUDE IN THE AHARONOV-BOHM GAUGE FIELD
}

\author{
Paola Giacconi ${ }^{(*)}{ }^{(1)}$, Fabio Maltoni ${ }^{(b)}{ }^{(2)}$, Roberto Soldati ${ }^{(\sharp)}$ \\ (*) Dipartimento di Matematica e Fisica, \\ Camerino, Italia \\ - sezione I.N.F.N. Bologna, Italia \\ (b) Dipartimento di Fisica, Pisa \\ - sezione I.N.F.N. Pisa, Italia \\ (\#) Dipartimento di Fisica "A. Righi", Bologna \\ - sezione I.N.F.N. Bologna, Italia
}

\begin{abstract}
A general expression for the scattering amplitude of nonrelativistic spinless particles in the Aharonov-Bohm gauge potential is obtained within the time independent formalism. The result is valid also in the backward and forward directions as well as for any choice of the boundary conditions on the wave function at the flux tube position.
\end{abstract}

DFUB/95 - 6

September 1995

(1) E-mail : giacconi, soldati@infn.bologna.it

(2) E-mail : maltoni@ipifidpt.difi.unipi.it 


\section{Introduction}

Since the appearence of the seminal paper [1] concerning the scattering on the plane of spinless charged particles in the field of an infinitely thin flux tube, the so called Aharonov-Bohm effect and related topics have been the sources of a very large number of investigations. Notwithstanding the really wide Literature on the subject, there are some few but relevant points, even within the originally considered scattering problem, which still deserve a more general mathematical formulation and, consequently, a more transparent physical interpretation, at least in our opinion. By the way the Aharonov-Bohm problem has recently gained a revival of interest, mainly because of its application to the anyon matter [2], to the cosmic strings [3] and to the planar gravity [4].

Basically there are two issues which still need a further analysis. The first one deals with the evaluation of the scattering amplitude in the backward and forward directions. To our knowledge this point has been firstly discussed in a very clear and rigorous way in ref. [5]. In this paper it is shown that, strictly speaking, the celebrated formula for the differential cross section holds true outside two opposite narrow cones, with common vertices at the point-like flux tube position, which contain the backward and forward directions respectively. As a matter of fact, on the one hand the asymptotic formulae, which allow to single out the scattering amplitude, are valid outside the above mentioned narrow cones. On the other hand, the alternative method described in ref. [5] to treat the problem, which makes use of a contour integral representation of the Bessel functions, is such that the separation between incident and scattered waves is no longer possible in the backward and forward directions; sic stantibus rebus a meaningful definition and eventual evaluation of the backward and forward scattering amplitudes are still lacking. There have been proposals [4],[6] to introduce some regularizations, in order to give a meaning to the sum over the partial phase shifts, which turns out to be meaningless as it stands. Then, however, delta-like singularities in the aforementioned critical directions unavoidably appear. As far as we understand, those singularities turn out to be awkward from the mathematical point of view [7] and, more important, they result to be a genuine effect of some specific choice 
(presumably not the most convenient) of a regularization procedure; consequently, it seems that their presence could likely be avoided.

The second issue is a concern of the choice of the boundary conditions for the wave function at the position of the infinitely thin flux tube. In the standard treatment the wave function is chosen to vanish at the flux tube position: this is a possible and simple choice, but far from being the most general one. Actually, a two real parameters family of boundary conditions exists, which stays into one-toone correspondence with the self-adjoint extensions of the quantum Hamiltonian operator [4],[8]. This fact entails that a more general formula for the differential cross section has to be considered, which generalizes the original Aharonov-Bohm famous result and reproduces the latter one for a special choice of the parameters of the self-adjoint extensions. As a by-product one can also get a clear understanding of the limits and the meaning of the Born approximation.

In this work we shall obtain a quite general formula for the scattering amplitude and the differential cross section, which includes the backward and forward directions as well as any choice of the boundary conditions on the wave function. The basic ingredients we shall develop here are the concept of the adiabatic switching of the interaction and the method of the analytic continuation of the scattering amplitudes. The paper is organized as follows. In sect. 2 we discuss the general framework and derive the formula for the scattering amplitude arising from the regular part of the wave function. In sect. 3 we treat the subspaces of the Hilbert space which are influenced by the choice of the boundary conditions at the flux tube position or, equivalently, by the self-adjoint extensions. In sect. 4 we discuss some consequences of our treatment, while the technical details leading to the main formulae are presented in the appendix.

\section{The scattering amplitude without the $S$ - and $P$-waves}

The standard non relativistic Hamiltonian for a spinless charged particle, with charge $e$ and mass $m$, moving on the plane in the presence of some infinitely thin flux tube located at the origin is given by

$$
H=\frac{1}{2 m}[\mathbf{p}-e \mathbf{A}(\mathbf{r})]^{2}
$$


where the Aharonov-Bohm (AB) gauge potentials is described as

$$
A_{j}(\mathbf{r})=\frac{\alpha}{e} \epsilon_{j k} \frac{x_{k}}{r^{2}} \quad j, k=1,2
$$

with $-1<\alpha<0$, which is the range we are here interested in. Setting $\epsilon_{12}=1$, the field strength can be written as

$$
F_{12}(\mathbf{r})=\frac{2 \pi|\alpha|}{e} \delta^{(2)}(\mathbf{r})
$$

If we split the Hamiltonian into free and interaction parts

$$
H(\mathbf{p}, \mathbf{r})=\frac{\mathbf{p}^{2}}{2 m}+V(\mathbf{p}, \mathbf{r})
$$

we obtain, going into polar coordinates $(r, \varphi)$,

$$
V\left(r, \frac{\partial}{\partial \varphi}\right)=\frac{\alpha}{2 m r^{2}}\left(-2 i \frac{\partial}{\partial \varphi}+\alpha\right)
$$

The solutions of the eigenvalue problem $H \psi_{k}^{A B}=E \psi_{k}^{A B}$, with $E=k^{2} / 2 m, k \geq 0$, corresponding to a particular set of stationary scattering states, are given by

$$
\psi_{k}^{A B}(r, \varphi)=(2 \pi)^{-1 / 2} \sum_{n=-\infty}^{+\infty} J_{|n+\alpha|}(k r) \exp \left\{i n \varphi-i \frac{\pi}{2}|n+\alpha|\right\}
$$

These eigenfunctions fulfil the following properties [1],[5]: namely,

$$
\begin{aligned}
& \text { i) } \psi_{k}^{A B}(r=0, \varphi)=0 ; \\
&\text { ii) } \left.\quad \psi_{k}^{A B}(r, \varphi) \sim \phi_{k}^{A B}(r, \varphi)+f^{A B}(k, \varphi) \frac{e^{i k r}}{\sqrt{2 \pi r}} \text { (large } r\right) ; \\
& \text { iii) }\left\langle\psi_{k}^{A B} \mid \psi_{p}^{A B}\right\rangle=\frac{1}{k} \delta(k-p) ;
\end{aligned}
$$

here the wave functions $\phi_{k}^{A B}(r, \varphi)$ do not correspond to eigenfunctions of the free Hamiltonian $H_{0}=\mathbf{p}^{2} / 2 m$, owing to the long range nature of the AB potential; they are given instead by the phase factors

$$
\phi_{k}^{A B}(r, \varphi)=\frac{1}{\sqrt{2 \pi}} \exp \{-i k r \cos \varphi-i \alpha \varphi\}
$$


It is worthwhile to stress that the asymptotic behaviour ii) holds true outside the sufficiently narrow cones $|\varphi|<\pi-\mathcal{O}\left[(k r)^{-1 / 2}\right]$ and, consequently, the phase factor $\phi_{k}^{A B}$ turns out to be single valued. The asymptotic behaviour of eq. (2.7b) can be obtained either from an integral representation [1],[9] for the exact solution of eq. (2.6), or from a contour integral representation of the Bessel functions [4],[5].

Since the eigenfunctions $\psi_{k}^{A B}$ are not normalizable, the scalar product in iii) is understood in the improper sense, i.e. in the tempered distributions topology

$$
\begin{aligned}
& \left\langle\psi_{k}^{A B} \mid \psi_{p}^{A B}\right\rangle= \\
& \mathcal{S}^{\prime}-\lim _{R \rightarrow \infty} \int_{0}^{R} r d r \int_{0}^{2 \pi} d \varphi\left[\psi_{k}^{A B}(r, \varphi)\right]^{*} \psi_{p}^{A B}(r, \varphi) .
\end{aligned}
$$

It turns out that the set $\left\{\tilde{\psi}_{k}^{A B} \mid \tilde{\psi}_{k}^{A B}(r, \varphi)=\sqrt{k} \psi_{k}^{A B}(r, \varphi), k \geq 0\right\}$ is a complete orthonormal set of improper scattering states of the Hilbert space $\mathcal{H}$. As a matter of fact the Hamiltonian (2.1), together with the boundary condition i), is a self-adjoint operator which does not admit bound states.

Now the main point. In order to define the scattering amplitude, there basically are two attitudes in the Literature. In the first one [1],[5] the amplitude is obtained from the asymptotic behaviour of the exact regular scattering solution of eq. (2.6). As already noticed, this point of view gives rise to the sum of a phase factor $\phi_{k}^{A B}=(2 \pi)^{-1 / 2} \exp \{-i(k r \cos \varphi+\alpha \varphi)\}$ and a scattered wave $\psi_{s c}=(2 \pi r)^{-1 / 2} f^{A B}(k, \varphi) \exp \{i k r\}$, the amplitude corresponding to the well known AB scattering amplitude [1]. However, as already noticed, the above mentioned asymptotic form holds true for $\varphi \neq l \pi, l \in \mathbf{Z}$; in other words, the above decomposition of the exact wave function, in the large- $r$ limit, strictly speaking loses its meaning in the backward and forward directions [5].

The second approach ${ }^{(*)}[4],[6]$ attempts to obtain the scattering amplitude

(*) To be specific, the authors of ref.s [4] actually treat the scattering on a spinning cone; nonetheless, the mathematical framework is in close correspondence to the case under consideration. 
as the sum of the partial amplitudes: namely,

$$
\begin{aligned}
f(k, \varphi) & =\frac{1}{\sqrt{2 \pi i k}} \sum_{n=-\infty}^{+\infty}\left(e^{2 i \delta_{n}}-1\right) e^{i n \varphi} \\
& =\lim _{\epsilon \rightarrow 0^{+}} \frac{1}{\sqrt{2 \pi i k}} \sum_{n=-\infty}^{+\infty}\left(e^{2 i \delta_{n}}-1\right) e^{i n \varphi-|n| \epsilon} \\
& =f_{r e g}(k, \varphi)
\end{aligned}
$$

Within this approach the regular solutions of the Schrödinger equation are written in the form

$$
\psi_{k}(r, \varphi)=\sum_{n=-\infty}^{+\infty} e^{i \delta_{n}+n \pi / 2} u_{n}(k r) e^{i n \varphi}
$$

whose asymptotic behaviours are provided by the usual expression

$$
\psi_{k}(r, \varphi) \sim \frac{1}{\sqrt{2 \pi}} e^{i k r \cos \varphi}+f_{r e g}(k, \varphi) \frac{e^{i k r}}{\sqrt{2 \pi r}} \quad(\text { large } r),
$$

where $f_{r e g}(k, \varphi)$ is formally given by the regularized quantity of eq. (2.10).

In so doing, however, the regularized amplitude appears to involve a mathematically poorly defined series of angular delta distributions in the forward and backward directions, also leading to troubles in the definition of the differential scattering cross section. Moreover, the remaining part of the amplitude actually reproduces the $\mathrm{AB}$ expression that is known to be correct only for $|\varphi|<\pi$. The authors of ref.s [4] suggest to move, in some sense, the delta-like contribution from the scattered wave to the incident plane wave in the limit of large- $r$, namely to redefine the decomposition of the wave function in the large- $r$ asymptote. Nevertheless, in order to implement this alternative decomposition, they eventually make use of some contour representation for the wave function and, therefore, they resort indeed to the first approach previously discussed. In conclusion, it seems that those angular delta distributions appear somewhat to be an artefact of the regularization of eq. (2.10), while the interpretation of the amplitude in the forward and backward directions remains admittedly still not quite clear [7].

The method we here develop is based on the standard integral equation satisfied by the scattering wave functions $\psi_{k}^{(+)}(r, \varphi)$. To this aim, it is convenient to split the wave function into the sum of the $S$-wave, carrying zero angular 
momentum, the $P$-wave of angular momentum $n=1$ and the rest: namely,

$$
\psi_{k}^{(+)}(r, \varphi) \equiv \chi_{0, k}^{(+)}(r)+\chi_{1, k}^{(+)}(r, \varphi)+\Psi_{k}^{(+)}(r, \varphi)
$$

where

$$
\Psi_{k}^{(+)}(r, \varphi)=\sum_{n=2}^{\infty} \frac{i^{n}}{\sqrt{2 \pi}} e^{i n \varphi-i \pi \alpha / 2} J_{n+\alpha}(k r)+\sum_{n=1}^{\infty} \frac{i^{n}}{\sqrt{2 \pi}} e^{-i n \varphi+i \pi \alpha / 2} J_{n-\alpha}(k r) .
$$

The $S$ - and $P$-waves will be discussed separately in the next section; the regular part $\Psi_{k}^{(+)}(r, \varphi)$ of the full eigenstate, which is different from the corresponding part in eq. (2.6), is constructed in such a way that the $n$-th partial wave has the usual large- $r$ asymptote, i.e.

$$
\begin{gathered}
\int_{0}^{2 \pi} \frac{d \varphi}{\sqrt{2 \pi}} e^{-i n \varphi}\left[\Psi_{k}^{(+)}(r, \varphi)-\frac{1}{\sqrt{2 \pi}} e^{i k r \cos \varphi}\right] \\
\sim \frac{e^{i k r}}{\sqrt{2 \pi i k r}}\left(e^{-i \pi \alpha}-1\right) \quad(\text { large } r),
\end{gathered}
$$

from the first sum in RHS of eq. (2.14) while

$$
\begin{gathered}
\int_{0}^{2 \pi} \frac{d \varphi}{\sqrt{2 \pi}} e^{i n \varphi}\left[\Psi_{k}^{(+)}(r, \varphi)-\frac{1}{\sqrt{2 \pi}} e^{i k r \cos \varphi}\right] \\
\sim \frac{e^{i k r}}{\sqrt{2 \pi i k r}}\left(e^{i \pi \alpha}-1\right) \quad(\text { large } r),
\end{gathered}
$$

from the second sum in the RHS of eq (2.14). By its very construction, the wave function $\Psi_{k}^{(+)}(\mathbf{r})$ satisfies the scattering integral equation

$$
\Psi_{k}^{(+)}(\mathbf{r})-\Phi_{k}(\mathbf{r})=\frac{m}{i} \int d^{2} \mathbf{r}^{\prime} H_{0}^{(1)}\left(k\left|\mathbf{r}-\mathbf{r}^{\prime}\right|\right) V\left(\mathbf{p}^{\prime}, \mathbf{r}^{\prime}\right) \Psi_{k}^{(+)}\left(\mathbf{r}^{\prime}\right),
$$

where $H_{0}^{(1)}$ is the first Hankel's function and

$$
\Phi_{k}(\mathbf{r})=(2 \pi)^{-1 / 2}\left[e^{i k r \cos \varphi}-J_{0}(k r)-i e^{i \varphi} J_{1}(k r)\right] .
$$

In order to extract from eq. (2.16) an expression for the related scattering amplitude, it is useful to introduce the concept of the adiabatic switching of the interaction, as it is customary in the perturbative field theory [10]. To this aim we 
consider, for instance, a smooth function $g_{\rho, R}(|\mathbf{r}|)$, with $\rho<R$ and $0 \leq g_{\rho, R}(r) \leq 1$, such that $g_{\rho, R}(r)=0, r \geq R$ and $g_{\rho, R}(r)=1,0 \leq r \leq \rho$, which represents the extent of switching on the interaction within a disk of radius $R$. In so doing, we can rewrite the RHS of eq. (2.16) in the form

$$
\begin{aligned}
& \Psi_{k}^{(+)}(\mathbf{r})-\Phi_{k}(\mathbf{r})= \\
& \lim _{\rho, R \rightarrow \infty} \frac{m}{i} \int d^{2} \mathbf{r}^{\prime} g_{\rho, R}\left(r^{\prime}\right) H_{0}^{(1)}\left(k\left|\mathbf{r}-\mathbf{r}^{\prime}\right|\right) V\left(\mathbf{p}^{\prime}, \mathbf{r}^{\prime}\right) \Psi_{k}^{(+)}\left(\mathbf{r}^{\prime}\right),
\end{aligned}
$$

the limit being understood in the $\mathcal{S}^{\prime}$-topology and taken at the very end. Now, as long as $\rho$ and $R$ are fixed, we can safely use the asymptotic formula for the Hankel's function, when $r$ is sufficiently large, leading to the result

$$
\begin{aligned}
& \Psi_{k}^{(+)}(\mathbf{r})-\Phi_{k}(\mathbf{r}) \sim \frac{-i m}{\sqrt{2 \pi i k}} \frac{e^{i k r}}{\sqrt{r}} \times \\
& \lim _{\rho, R \rightarrow \infty} \int d^{2} \mathbf{r}^{\prime} g_{\rho, R}\left(r^{\prime}\right) \exp \left\{-i \mathbf{k} \cdot \mathbf{r}^{\prime}\right\} V\left(\mathbf{p}^{\prime}, \mathbf{r}^{\prime}\right) \Psi_{k}^{(+)}\left(\mathbf{r}^{\prime}\right) \quad(\text { large } r)
\end{aligned}
$$

where $\mathbf{k} \equiv k \mathbf{r} / r$ is the momentum of the incident plane wave. Taking eventually the limits $\rho, R \rightarrow \infty$, we finally obtain a definition of the scattering amplitude, apart from the $S$ - and $P$ - waves, given by

$$
\begin{aligned}
F_{k}^{(+)}(\varphi) & =\frac{2 \pi \alpha}{i \sqrt{2 \pi i k}} v(\varphi, \alpha) \times \\
& \sum_{n=2}^{\infty} \int_{0}^{\infty} \frac{d x}{x}\left[e^{-i n \varphi-i \pi \alpha / 2} J_{n+\alpha}(x) J_{n}(x)+e^{i n \varphi+i \pi \alpha / 2} J_{n-\alpha}(x) J_{n}(x)\right] \\
& +\frac{2 \pi \alpha}{i \sqrt{2 \pi i k}} v(\varphi, \alpha) \int_{0}^{\infty} \frac{d x}{x} e^{i \varphi+i \pi \alpha / 2} J_{1-\alpha}(x) J_{1}(x)
\end{aligned}
$$

where $v(\varphi, \alpha) \equiv \alpha+2 i(\partial / \partial \varphi)$ and $x \equiv k r^{\prime}$. It should be gathered that the interaction can be fully switched on (i.e. $\rho, R \rightarrow \infty)$, provided the order of the operations is fixed as in eq. (2.20): that means, first radial integration, then sum over angular momenta and finally derivative with respect to the angle. In so doing, the amplitude is perfectly well defined, without need of any ad hoc regularization. We remark that, in the case of short-range interactions, the adiabatic limit in eq. (2.19) is trivial and the standard textbook formula is immediately recovered. Moreover, the above definition (2.19) is equivalent to the one which is obtained from the 
usual asymptotic behaviour of the scattering wave eigenfunctions whereas, for a long-range potential, this is not exactly true in general, as the present analysis indeed shows.

In the specific $\mathrm{AB}$ scattering problem eq. (2.20) entails $(l \in \mathbf{Z})$

$$
F_{k}^{(+)}(\varphi)=\frac{1}{\sqrt{2 \pi i k}}\left[1-\cos \pi \alpha-e^{-i \varphi}\left(e^{-i \pi \alpha}-1\right)-\sin \pi \alpha \cot \frac{\varphi}{2}\right]
$$

when $\varphi \neq 2 l \pi$. Furthermore, the following expression holds true in the forward direction: namely,

$$
F_{k}^{(+)}(\varphi=2 l \pi)=\frac{1}{\sqrt{2 \pi i k}}[2(1-\cos \pi \alpha)+i(1+\alpha) \sin \pi \alpha] .
$$

The details are given in the appendix; here we would like to add some remarks. Firstly we see that eq. (2.21) is nothing but the AB amplitude, up to the $S$ - and $P$ wave contributions; furthermore, from eq. (2.19), it is apparent that the incoming wave is a standard plane wave and no angular delta distributions ever appear. This in turn entails that, at variance with the formula (2.7b), the amplitude can be singled out and evaluated, by means of a unique analytic continuation, also in the backward and forward directions. Finally, we notice that eq. (2.22) could also be reinterpreted in the framework of the time dependent approach, as proposed in ref. [9].

\section{The $S$ - and $P$-wave scattering amplitudes}

In order to properly treat the $S$ - and $P$-waves, we have to consider two facts. The first one is to recognize that, in general, regularity at $r=0$ is too a strong requirement for the eigenfunctions of zero and one angular momentum. As a matter of fact, self-adjointness of the Schrödinger Hamiltonian in eq. (2.1) can be indeed fulfilled even by singular $S$ - and $P$-wave functions (although locally square integrable around the origin) [8]. Consequently, the vanishing boundary condition at the origin as in eq. (2.7a) does not appear to be a necessary requirement, for it has to be suitably generalized. The second one is that, owing to the presence of a singular part in the above mentioned partial waves, the corresponding scattering amplitude has to be necessarily obtained from the large- $r$ asymptote of some $S$ - and 
$P$-waves satisfying the most general boundary conditions we shall specify below; actually, a definition of those partial waves amplitudes following the method of the previous section is no longer available, due to the infrared divergencies in the integral of eq. (2.20). According to the Von Neumann theory of the deficiency indices [11], one obtains the most general behaviour at the origin of the wave function: namely,

$$
\psi(\mathbf{r}) \sim \sum_{n=0,1} C_{n} r^{\gamma(n)} \quad(\text { small } r)
$$

where $C_{n}$ are some $r$-independent quantities and $-1<\gamma(n)<0$.

With the boundary condition established in eq. (3.1) the $S$ - and $P$ - scattering wave functions become

$$
\begin{aligned}
\chi_{0, k}^{(+)}(r) & =\frac{\exp (i \pi \alpha / 2)}{\sqrt{2 \pi}\left(1+i \tan \pi \mu_{0}\right)}\left[J_{-\alpha}(k r)+\tan \pi \mu_{0} N_{-\alpha}(k r)\right], \\
\chi_{1, k}^{(+)}(r, \varphi)= & -\frac{\exp [-i(\pi / 2)(1+\alpha)+i \varphi]}{\sqrt{2 \pi}\left(1+i \tan \pi \mu_{1}\right)} \\
& \times\left[J_{1+\alpha}(k r)+\tan \pi \mu_{1} N_{1+\alpha}(k r)\right],
\end{aligned}
$$

where

$$
\begin{aligned}
\tan \pi \mu_{0}(k) & \equiv \frac{\sin \pi \alpha}{\cos \pi \alpha-\left(k^{2} / 2 m E_{0}\right)^{\alpha}}, \\
\tan \pi \mu_{1}(k) & \equiv \frac{\sin (-\pi \alpha)}{\cos \pi \alpha+\left(k^{2} / 2 m E_{1}\right)^{-(1+\alpha)}},
\end{aligned}
$$

$E_{0}, E_{1}$ being bound state energies for the angular momenta $n=0$ and $n=1$ respectively. Actually, for any choice of the self-adjoint extensions corresponding to fixed values of the parameters $\mu_{0} \in \mathbf{R}, \mu_{1} \in \mathbf{R}$, there exist solutions of the equations $H B_{0}(r)=-E_{0} B_{0}(r)$, for $n=0$ and $E_{0}>0, H B_{1}(r, \varphi)=-E_{1} B_{1}(r, \varphi)$, for $n=1$ and $E_{1}>0$. The normalized eigenfunctions are

$$
\begin{aligned}
B_{0}(r) & =\left(\kappa_{0} / \pi\right) \sqrt{\sin (-\pi \alpha)} K_{\alpha}\left(\kappa_{0} r\right), \\
B_{1}(r, \varphi) & =\left(\kappa_{1} / \pi\right) \sqrt{\sin \pi(1+\alpha)} K_{1+\alpha}\left(\kappa_{1} r\right) e^{i \varphi}, \\
\kappa_{0(1)} & \equiv \sqrt{2 m E_{0(1)}},
\end{aligned}
$$

which turn out to be orthogonal to the scattering eigenfunctions. In order to obtain regular scattering wave functions from eq.s (3.2) one has to take the limits 
$E_{0} \rightarrow \infty$ and $E_{1} \rightarrow \infty$ that give $\tan \pi \mu_{0}=\tan \pi \mu_{1}=0$. It has to be gathered that, as already noticed, in the above limits the bound states disappear as the corresponding wave functions (3.4) indeed identically vanish. Accordingly, the regular scattering wave functions are a complete orthonormal set which diagonalize the self-adjoint invertible quantum Hamiltonian and, safely, there do not appear bound states with infinitely negative energies, whose physical interpretation turned out to be troublesome.

On the other hand, taking the limits $E_{0} \rightarrow 0$ and $E_{1} \rightarrow 0$, from which $\tan \pi \mu_{0}=-\tan \pi \mu_{1}=\tan \pi \alpha$, one is led to the purely singular eigenfunctions. Moreover, in the absence of AB potential $(\alpha \rightarrow 0)$, eq.s (3.2) give scattering wave functions in the presence of a purely contact interaction; we have to stress that, in this limit, only the $S$ - wave exhibits a singular part: namely,

$$
\begin{aligned}
& \lim _{\alpha \rightarrow 0} \tan \pi \mu_{0}=-\frac{\pi}{\ln \left(k^{2} / 2 m E_{0}\right)}, \\
& \lim _{\alpha \rightarrow 0} \tan \pi \mu_{1}=0 .
\end{aligned}
$$

In other words we can say that the $P$ - wave is not influenced by the pure $\delta$-like potential. Now we are ready to compute the scattering amplitudes for $S$ - and $P$ waves from their large- $r$ behaviour. They read

$$
\begin{aligned}
& f_{0}^{(+)}\left(k ; E_{0}\right)=\frac{1}{\sqrt{2 i \pi k}}\left[e^{i \pi \alpha}\left(\cos 2 \pi \mu_{0}-i \sin 2 \pi \mu_{0}\right)-1\right], \\
& f_{1}^{(+)}\left(k, \varphi ; E_{1}\right)=\frac{e^{-i \varphi}}{\sqrt{2 i \pi k}}\left[e^{-i \pi \alpha}\left(\cos 2 \pi \mu_{1}-i \sin 2 \pi \mu_{1}\right)-1\right] .
\end{aligned}
$$

It should be noticed that, from eq.s (3.6), a nonvanishing contribution to the scattering amplitude arises also in the backward and forward directions. It is also interesting to consider the limit $\alpha \rightarrow 0$ in eq.s (3.6): namely,

$$
\begin{aligned}
& \lim _{\alpha \rightarrow 0} f_{0}^{(+)}\left(k ; E_{0}\right)=\frac{1}{\sqrt{2 i \pi k}}\left[\exp \left\{2 i \arctan \frac{\pi}{\ln \left(2 m E_{0} / k^{2}\right)}\right\}-1\right], \\
& \lim _{\alpha \rightarrow 0} f_{1}^{(+)}\left(k, \varphi ; E_{1}\right)=0
\end{aligned}
$$

which confirms that the $P$ - wave is not influenced by a $\delta$-like potential. 
In order to obtain the usual $\mathrm{AB}$ scattering amplitude we have to consider the (regular) limits $E_{0} \rightarrow \infty$ and $E_{1} \rightarrow \infty$ of eq.s (3.6); this entails

$$
\begin{aligned}
& \lim _{E_{0} \rightarrow \infty} f_{0}^{(+)}\left(k ; E_{0}\right)=\frac{1}{\sqrt{2 i \pi k}}\left(e^{i \pi \alpha}-1\right), \\
& \lim _{E_{1} \rightarrow \infty} f_{1}^{(+)}\left(k, \varphi ; E_{1}\right)=\frac{e^{-i \varphi}}{\sqrt{2 i \pi k}}\left(e^{-i \pi \alpha}-1\right) ;
\end{aligned}
$$

by adding both the above expression to eq. (2.21a), one easily reconstruct the well known AB formula.

It is also easy to recover the phase shifts corresponding to the $\mathrm{AB}$ and purely contact $\delta$-like potentials $(\alpha \rightarrow 0)$ for angular momenta $n=0$ and $n=1$ respectively: they read,

$$
\begin{aligned}
& \delta_{0}\left(k, \alpha ; E_{0}\right)= \\
& \frac{\pi \alpha}{2}-\arctan \frac{\sin \pi \alpha}{\cos \pi \alpha-\left(k^{2} / 2 m E_{0}\right)^{\alpha}} \stackrel{\alpha \rightarrow 0}{\longrightarrow} \arctan \frac{\pi}{\ln \left(2 m E_{0} / k^{2}\right)} ; \\
& \delta_{1}\left(k, \alpha ; E_{1}\right)=-\frac{\pi \alpha}{2}+\arctan \frac{\sin \pi \alpha}{\cos \pi \alpha-\left(k^{2} / 2 m E_{1}\right)^{-(\alpha+1)}} .
\end{aligned}
$$

It should be noticed that eq.s (3.9) correctly reproduce all the limits $\alpha \rightarrow 0$ and $E_{0}, E_{1} \rightarrow \infty$, at variance with the incorrect formula of the first paper in ref. [8].

\section{Discussion}

In the presence of a general behaviour at the origin, as expressed by the relation (3.1), the set $\left\{\tilde{\psi}_{k}^{A B} \mid k \geq 0\right\}$ no longer represents a (improper) basis in the Hilbert space. We have instead to select, for instance, the following complete orthonormal family $(k \geq 0)$

$$
\begin{aligned}
\chi_{k, n}(r, \varphi) & =\sqrt{\frac{k}{2 \pi}} J_{|n+\alpha|}(k r) \exp \{i n \varphi\} \quad n \neq 0,1 ; \\
\chi_{k, 0}\left(r ; E_{0}\right) & =\sqrt{\frac{k}{2 \pi}}\left\{\cos \pi \mu_{0}(k) J_{|\alpha|}(k r)+\sin \pi \mu_{0}(k) N_{|\alpha|}(k r)\right\} ; \\
\chi_{k, 1}\left(r, \varphi ; E_{1}\right) & =\sqrt{\frac{k}{2 \pi}}\left\{\cos \pi \mu_{1}(k) J_{1-|\alpha|}(k r)\right. \\
& \left.+\sin \pi \mu_{1}(k) N_{1-|\alpha|}(k r)\right\} \exp \{i \varphi\} \\
B_{0}(r) & =\left(\kappa_{0} / \pi\right) \sqrt{\sin (-\pi \alpha)} K_{\alpha}\left(\kappa_{0} r\right) ; \\
B_{1}(r, \varphi) & =\left(\kappa_{1} / \pi\right) \sqrt{\sin \pi(1+\alpha)} K_{1+\alpha}\left(\kappa_{1} r\right) e^{i \varphi} .
\end{aligned}
$$


In particular, according to the fundamental theorem for self-adjoint operators, the following completeness relation must hold: namely,

$$
\begin{aligned}
& \int_{0}^{\infty} \frac{k d k}{2 \pi}\left\{\cos ^{2} \pi \mu_{0}(k) J_{|\alpha|}(k r) J_{|\alpha|}\left(k r^{\prime}\right)+\sin ^{2} \pi \mu_{0}(k) N_{|\alpha|}(k r) N_{|\alpha|}\left(k r^{\prime}\right)\right. \\
& \left.+(1 / 2) \sin 2 \pi \mu_{0}(k)\left(J_{|\alpha|}(k r) N_{|\alpha|}\left(k r^{\prime}\right)+J_{|\alpha|}\left(k r^{\prime}\right) N_{|\alpha|}(k r)\right)\right\}=\delta^{(2)}\left(\mathbf{r}-\mathbf{r}^{\prime}\right),
\end{aligned}
$$

and the analogous relation for the $n=1$ angular momentum Hilbert subspace. The above completeness relations turn out to be quite useful, in order to construct integral kernels of operators commuting with the Hamiltonian; furthermore, such a kind of resolutions of the identity become powerful tools, for instance, in the calculation of physical quantities such as the second virial coefficient of the anyon gas [12] or the anomaly of the axial current in the spinning case [13]. It should also be noticed that the normalization of the improper scattering states in eq.s (3.2) is, instead, rather cumbersone in general; on the other hand they exhibit, by their very construction, a standard asymptotic behaviour. These features are in contrast with the properties of the regular scattering states $\tilde{\psi}_{k}^{A B}(r, \varphi)$, which fulfil standard normalization (see eq. (2.7c)) but non standard asymptotic form (see eq. (2.7b)).

Another interesting aspect of the present approach is that it allows a direct comparison with the Born approximation, as it is apparent from eq. (2.20). As a matter of fact, by adding the RHS of eq. $(3.8 \mathrm{~b})$, which represents the contribution of the regular $P$-wave, to the amplitude in eq. (2.21) we easily obtain, up to the first order in the coupling $\alpha$, the Born approximation [14], i.e.

$$
f_{\text {Born }}^{(+)}(k, \varphi)=\frac{|\alpha| \pi}{\sqrt{2 \pi i k}} \cot \frac{\varphi}{2} .
$$

Actually, the above expression is fully determined by the (regular) components of the plane waves with non vanishing angular momentum, since the $S$-wave leads to a divergent contribution but $\mathcal{O}\left(\alpha^{2}\right)$. This means that, within the Born approximation, contact-like interaction can not be taken into account; as a consequence the expansion of the full amplitude

$$
f_{k}^{(+)}\left(\varphi ; E_{0}, E_{1}\right) \equiv F_{k}^{(+)}(\varphi)+f_{0}^{(+)}\left(k, \varphi ; E_{0}\right)+f_{1}^{(+)}\left(k, \varphi ; E_{1}\right)
$$

up to the first order in $\alpha$, obviously does not lead to eq. (4.3). 
As a final comment, we would like to observe that the full amplitude in eq. (4.4) when $\varphi=2 l \pi, l \in \mathbf{Z}$, gives rise to a finite differential cross section in the forward direction. This means that, even within the time-independent approach, the concept of the adiabatic switching of the interaction allows a complete proper description of the $\mathrm{AB}$ physics at variance with the usual phase shifts analysis. Notice that the inadequacy of the phase shifts approach in the treatment of the AB problem has led the author of ref. [9] to develop the time dependent approach which, however, appears to be more involved than the time independent one, at least in our opinion. Furthermore, in that paper an explicit expression is not given for the forward scattering amplitude, but only arguments concerning the scattering of some wave packets.

We also notice that the present analysis might be generalized to the spinning case as well as to the scattering of particles in a $2+1$ dimensional space-time in the presence of gravitational fields.

\section{Acknowledgments:}

This work has been partially supported by a grant from Ministero della Università e della Ricerca Scientifica e Tecnologica - quota 40\% .

\section{Appendix}

Here we want to show how eq.s (2.21-22) can be derived. The starting point is the scattering amplitude, defined by eq. (2.20), whose RHS we want here to evaluate it in a straightforward way. Taking eq.s (2.5) and (2.14) into account, after integration over $\varphi^{\prime}$ and rescaling of the integration variable $k r^{\prime} \equiv x$ respectively, 
we obtain

$$
\begin{aligned}
F_{k}^{(+)}(\varphi) & =\frac{2 \pi \alpha}{i \sqrt{2 \pi i k}} v(\varphi, \alpha) \times \\
& \sum_{n=2}^{\infty} \int_{0}^{\infty} \frac{d x}{x}\left[e^{-i n \varphi-i \pi \alpha / 2} J_{n+\alpha}(x) J_{n}(x)+e^{i n \varphi+i \pi \alpha / 2} J_{n-\alpha}(x) J_{n}(x)\right] \\
& +\frac{2 \pi \alpha}{i \sqrt{2 \pi i k}} v(\varphi, \alpha) \int_{0}^{\infty} \frac{d x}{x} e^{i \varphi+i \pi \alpha / 2} J_{1-\alpha}(x) J_{1}(x) \\
& =\frac{2}{i \sqrt{2 \pi i k}} \sin \left(\frac{\pi \alpha}{2}\right) v(\varphi, \alpha)\left[\sum_{n=2}^{\infty} \frac{e^{-i \pi \alpha / 2-i n \varphi}}{n+(\alpha / 2)}-\sum_{n=1}^{\infty} \frac{e^{i \pi \alpha / 2+i n \varphi}}{n-(\alpha / 2)}\right]
\end{aligned}
$$

where $v(\varphi, \alpha) \equiv \alpha+2 i(\partial / \partial \varphi)$ and $x \equiv k r^{\prime}$. We want to stress that all the above expressions are perfectly well defined, provided the operator $v(\alpha, \varphi)$ is applied after summing the series; consequently, taking the above specified order of operations carefully into account (integration, sum, action of the $v$-operator), there is no need of any regularization. As a matter of fact, from the basic formula [15]:

$$
\sum_{n=0}^{\infty} \frac{e^{-i n \varphi}}{n+z}=\beta(z)+\frac{e^{i z \varphi}}{2} \int_{\varphi}^{\pi} d t e^{-i z t}\left(\cot \frac{t}{2}+i\right)
$$

we recover, after addition of the partial waves contributions from eq.s (3.8), the well known AB scattering amplitude

$$
f_{A B}^{(+)}(k, \varphi)=\frac{1}{\sqrt{2 \pi i k}} \sin \pi|\alpha|\left(\cot \frac{\varphi}{2}-i\right), \quad \varphi \neq 2 l \pi
$$

it should be enphasized that, at variance with previous derivations [1],[5], within the present framework eq. (A.3) holds true even in the backward direction.

Now the key point is to realize that eq. (A.1) allows a unique analytic continuation, depending upon a complex variable $s$, which admits a well defined limit at the physical value $s=1$, even in the forward direction $\varphi=2 l \pi$. To this aim, let us introduce the analytically continued amplitude: namely,

$$
\begin{aligned}
F_{k}^{(+)}(\varphi ; s) & \equiv \frac{2}{i \sqrt{2 \pi i k}} \sin \left(\frac{\pi \alpha}{2}\right) \times \\
& \left\{e^{i \pi \alpha / 2}\left(-\frac{\alpha}{2}\right)^{1-s}-e^{-i \pi \alpha / 2}\left[e^{-i \varphi}\left(1+\frac{\alpha}{2}\right)^{1-s}+\left(\frac{\alpha}{2}\right)^{1-s}\right]\right. \\
& \left.+\sum_{n=0}^{\infty}\left[\frac{e^{-i \pi \alpha / 2-i n \varphi}}{(n+(\alpha / 2))^{s-1}}-\frac{e^{i \pi \alpha / 2+i n \varphi}}{(n-(\alpha / 2))^{s-1}}\right]\right\}
\end{aligned}
$$


We stress that, within the strip $R e s \geq 2$, the $v$-operator can be freely interchanged with the series since, now, the physical limit $s \rightarrow 1$ will be performed at the very end. In the case $\varphi \neq 2 l \pi(l \in \mathbf{Z})$, eq. (A.4) becomes

$$
\begin{aligned}
F_{k}^{(+)}(\varphi ; s) & =\frac{2 i}{\sqrt{2 \pi i k}} \sin \left(\frac{\pi \alpha}{2}\right)\left\{e^{i \pi \alpha / 2}\left[\Phi\left(e^{i \varphi}, s-1,-\alpha / 2\right)-\left(-\frac{\alpha}{2}\right)^{1-s}\right]\right. \\
& \left.-e^{-i \pi \alpha / 2}\left[\Phi\left(e^{-i \varphi}, s-1, \alpha / 2\right)-e^{-i \varphi}\left(1+\frac{\alpha}{2}\right)^{1-s}-\left(\frac{\alpha}{2}\right)^{1-s}\right]\right\} ;
\end{aligned}
$$

therefore, using the analytic extension of the function $\Phi[16]$, we get

$$
\begin{aligned}
\Phi(z, s=1, v) & =\frac{1}{z^{v}}\left[\frac{1}{\ln (1 / z)}-\sum_{n=1}^{\infty} \frac{1}{n !} B_{n}(v)(\ln z)^{n-1}\right] \\
& =\frac{1}{1-z}, \quad|\ln z|<2 \pi
\end{aligned}
$$

where $B_{n}(v)$ denote the Bernoulli polynomials. From the last formula we can easily recover eq. (2.21): namely,

$$
\begin{aligned}
F_{k}^{(+)}(\varphi) & \equiv \lim _{s \rightarrow 0^{+}} F_{k}^{(+)}(\varphi ; s)=\frac{2 i}{\sqrt{2 \pi i k}} \sin \left(\frac{\pi \alpha}{2}\right) \times \\
& {\left[e^{i \pi \alpha / 2}\left(\frac{1}{1-e^{i \varphi}}-1\right)-e^{-i \pi \alpha / 2}\left(\frac{1}{1-e^{-i \varphi}}-e^{-i \varphi}-1\right)\right] } \\
& =\frac{1}{\sqrt{2 \pi i k}}\left[1-\cos \pi \alpha-e^{-i \varphi}\left(e^{-i \pi \alpha}-1\right)-\sin \pi \alpha \cot \frac{\varphi}{2}\right]
\end{aligned}
$$

Moreover, in the case $\varphi=2 l \pi$, the forward scattering amplitude of eq. (A.4) can be defined by means of the Hurwitz Zeta Function $\zeta(s, q)[16]$ : actually,

$$
\begin{aligned}
F_{k}^{(+)}(\varphi=2 l \pi ; s) & \equiv \frac{2 i}{\sqrt{2 \pi i k}} \sin \left(\frac{\pi \alpha}{2}\right)\left\{e^{i \pi \alpha / 2}\left[\zeta(1-s,-\alpha / 2)-\left(-\frac{\alpha}{2}\right)^{1-s}\right]\right. \\
& \left.-e^{-i \pi \alpha / 2}\left[\zeta(1-s, \alpha / 2)-\left(1+\frac{\alpha}{2}\right)^{1-s}-\left(\frac{\alpha}{2}\right)^{1-s}\right]\right\}
\end{aligned}
$$

which easily leads to eq. (2.22), since

$$
\begin{aligned}
& F_{k}^{(+)}(\varphi=2 l \pi) \equiv \lim _{s \rightarrow 1} F_{k}^{(+)}(\varphi=2 l \pi ; s)= \\
& \frac{2}{i \sqrt{2 \pi i k}} \sin \left(\frac{\pi \alpha}{2}\right)\left\{e^{-i \pi \alpha / 2}\left[B_{1}(-\alpha / 2)+1\right]-e^{i \pi \alpha / 2}\left[B_{1}(\alpha / 2)+2\right]\right\}
\end{aligned}
$$




\section{References}

[1] Y. Aharonov and D. Bohm, Phys. Rev. 115 (1959) 485.

[2] F. Wilczek, "Fractional Statistics and Anyon Superconductivity", World Publishing, Singapore (1990);

P. de Sousa Gerbert, Int. Jour. Mod. Phys. A 6 (1991) 173;

R. Iengo and K. Lechner, Phys. Rep. 213 (1992) 180;

S. Forte, Rev. Mod. Phys. A 7 (1992) 1025.

[3] A. Vilenkin, Phys. Rep. 121 (1985) 263 and references therein.

[4] S. Deser and R. Jackiw, Comm. Math. Phys. 118 (1988) 495;

P. de Sousa Gerbert and R. Jackiw, Comm. Math. Phys. 124 (1989) 229.

[5 ] M. V. Berry, R. G. Chambers, M. D. Large, C. Upstill and J. C. Walmsley, Eur. J. Phys. 1 (1980) 154.

[6 ] S. N. M. Ruijsenaars, Ann. of Phys. 146 (1983) 1.

[7] C. R. Hagen, Phys. Rev. D 41 (1990) 2015.

[8 ] C. Manuel and R. Tarrach, Phys. Lett. B 268 (1991) 222.

J. Grundberg, T. H. Hansson, A. Karlhede and J. M. Leinaas, Mod. Phys. Lett. B 5 (1991) 539.

[9 ] D. Stelitano, preprint MIT-CTP\# 2383 (1994).

[10 ] N. N. Bogoliubov and D. V. Shirkov, "Introduction to the theory of quantized fields", John Wiley \& Sons, New York (1959), p. 198.

[11 ] S. Albeverio, F. Gesztesy, R. Hoegh-Krohn and H. Holden, " Solvable Models in Quantum Mechanics", Springer-Verlag, New York (1988);

M. Reed and B. Simon, "Fourier Analysis and Self-Adjointness", Academic Press, Orlando (1987).

[12 ] A. Comtet and S. Ouvry, Phys. Lett. B 225 (1989) 272;

A. Comtet, Y. Georgelin and S. Ouvry, J. Phys. A 22 (1989) 3917.

[13 ] P. Giacconi, S. Ouvry and R. Soldati, Phys. Rev. D 50 (1994) 5358.

[14] E. Corinaldesi and F. Rafeli, Am. J. Phys. 46 (1978) 1185.

[15] A. P. Prudnikov, Yu. A. Brychkov and O. I. Marichev, "Integrals and series", Gordon and Breach Science Publishers, New York (1992). 
[16 ] I. S. Gradshteyn and I. M. Ryzhik, "Table of integrals series and products", Academic Press, San Diego (1979). 